\title{
Polymeric Biomaterials for Gene and Drug Delivery
}

\author{
David Oupicky
}

Received: 10 October 2010 / Accepted: 11 October 2010 / Published online: 22 October 2010

(C) Springer Science+Business Media, LLC 2010

Polymeric biomaterials for gene and drug delivery remain an active area of research. In particular, the development of cationic polymers for gene delivery continues to be the focus of intense activity as development of new synthetic strategies and design of new polycations remain at the heart of the progress in the field of gene delivery. As a result of the emphasis on synthesis of new polymers, we now have hundreds, if not thousands, of polycations available for gene delivery. Advances in controlled polymerization methods helped to overcome many limitations of polymers related to their high polydispersity and poor control of polymer architecture. The advances in synthesis in turn resulted in improved modular design of polyplexes that effectively combine multiple functionalities into a single delivery system.

In addition to the advances in polycation synthesis, there has been increased emphasis on improving our understanding of how polyplexes interact with living cells. We now have deeper understanding of the factors that govern toxicity and subcellular trafficking of polyplexes. This knowledge has been successfully applied to the design of safer and more efficient gene delivery vectors. Despite significant progress, many delivery challenges remain to be addressed to make therapeutic nucleic acids a widespread clinical modality. The most important one that has eluded the field for so long is a robust and reproducible method to deliver nucleic acids systemically. One of the reasons for this failure is that we lack the predictive tools to select candidates that would succeed systemically based on in vitro data. Many highly innovative delivery designs that work beautifully in cell culture fail completely in vivo.

D. Oupicky $(\square)$

Wayne State University,

Detroit, Michigan, USA

e-mail: oupicky@wayne.edu
This theme section of Pharmaceutical Research brings together several contributions that showcase the possibilities of synthetic nucleic acid delivery vectors in pulmonary and brain delivery and that illustrate the potential of modular vector design for improved delivery efficacy.

Localized delivery of nucleic acids onto mucosal surfaces is an attractive alternative to systemic delivery. The localized delivery reduces nuclease degradation and the need to avoid hepatic clearance of the particles. Nielsen et al. describe the preparation and application of an aerosolized formulation of chitosan nanoparticles for improved pulmonary siRNA delivery and gene silencing in mice. This work serves to provide a platform for effective pulmonary delivery and gene silencing of RNAi therapeutics with potential use in preclinical studies of respiratory disease treatment.

Brain is one of the most challenging delivery targets for nucleic acid delivery vectors. Zhang et al. describe an innovative modular delivery system based on intercalating multifunctional copolymers for gene delivery to brain capillaries. This delivery system shows improved transfection efficacy in slow-dividing cells compared to control PEI. Systemic intravenous injection of the delivery vector carrying DNA producing siRNA against drug efflux transporter protein MRP4 enabled enhanced uptake of the antiviral drug AZT in the brain by 230-270\%.

Raviña et al. report the development of another modular system for delivery of DNA and siRNA based on nanoparticles consisting of PEG, chitosan, and hyaluronic acid. The authors demonstrate that the particles can be easily adapted for the delivery of different types of nucleic acids. The particles were able to efficiently inhibit Snaill transcription factor, thus confirming the potential of the developed nanoparticles for siRNA-based anticancer therapies. 
Combinations of two or more chemotherapeutic agents with pharmacodynamically synergistic or additive effects are effectively used in cancer therapy. Combining traditional small molecule drugs with nucleic acid-based drugs, such as genes and siRNA, provides additional flexibility in selecting synergistic or additive targets in combination therapies. Although drug/gene combinations have been investigated and used in the treatment of cancer, no proven methods of systemic delivery are available to achieve simultaneous drug/gene delivery to tumors. In the last report, Bhattarai et al. describe the preparation of hybrid biomaterials based on mesoporous silica nanoparticles and polycations suitable for simultaneous delivery of drug/gene and drug/siRNA combinations. The authors show that the particles are able to successfully deliver plasmid DNA and siRNA in cell culture. Loading the particles with chloroquine further enhances transfection activity of both investigated nucleic acid types, confirming the suitability of the hybrid particles for combination drug/gene and drug/ siRNA delivery.

\section{INTERVIEW WITH DR. DAVID OUPICKY}

What do you think holds the key to your success as a pharmaceutical scientist?

A pharmaceutical scientist must have a broad perspective and at least basic understanding of both the physical and biological aspects of his or her work. After all, what comes out of pharmaceutical research has to work in a complex biological environment.

What do you consider to be your key research accomplishments? From our recent work, it would have to be our research on bioreducible polycations and their use for gene delivery, both in soluble form as polyplexes and in the form of thin DNA films. We have developed several different types of bioreducible polycations, ranging from peptides to polymers synthesized by living radical polymerization. We have shown the importance of intracellular and exofacial plasma membrane redox environment for activity and toxicity of these delivery systems. From our earlier work, I think the key discovery was the importance of combining steric and lateral stability of polyplexes for improving their pharmacokinetics and biodistribution.

\section{What was the turning point in your career?}

It is usually difficult to identify a single turning point in someone's career, but I think mine was attending a departmental seminar of Dr. Karel Ulbrich on HPMA copolymers for drug delivery at the Prague Institute of Chemical Technology (ICT). At that time, I was a first year Ph.D. student at ICT working in the field of high- performance polymers, blissfully unaware of the entire exciting field of biomedical polymers. After a short intermezzo at Polytechnic University in Brooklyn, I decided to leave ICT and joined Karel's lab at the Institute of Macromolecular Chemistry. Even though it cost me at least a year on the way to my Ph.D., it was a great career decision.

Which individuals have most influenced your research career?

Two people that influenced my research career most were my Ph.D. and postdoc mentors, Karel Ulbrich and Len Seymour, respectively. I have learned a lot from them about the importance of working with scientists in other fields and how vital it is to consider biological aspects of drug and gene delivery from early stages of development.

Pharmaceutical scientists are faced with the dilemma of having to publish in biomedical or basic science journals. Does this mean cutting-edge science will not likely be featured in journals like Pharmaceutical Research? That is an issue faced by most specialized journals, and it is ultimately up to the people in the field to nurture and support such journals by submitting their good work there. I think Pharmaceutical Research is in a good position in that regard.

Where is the field of Biomaterials for Gene and Drug Delivery going, and how do the articles in this theme section fill the gap?

The field is evolving in several directions. On the chemistry side, we have available increasingly sophisticated methods of polymer synthesis that allow us to synthesize betterdefined polymers and polymer architectures. We now have available libraries of cationic polymers that allow us to better map the chemical space. On the delivery side, the focus is on improving understanding of the biological underpinnings of the vector behavior, especially when it comes to intracellular trafficking. The articles in the theme section fill the gap we have in the development of vectors for in vivo delivery of nucleic acids and in the design of modular delivery vectors that successfully combine multiple functionalities.

What are the challenges for Biomaterials for Gene and Drug Delivery, and how can they be overcome?

Despite the fact that hundreds, if not thousands, of new polymers and lipids have been developed, the challenges remain disappointingly similar to what we faced 10 or 15 years ago. We are still unable to deliver nucleic acids reliably in vivo. We have seen incremental advances due to improved methods of controlled polymer synthesis and the emergence of combinatorial polymer and lipid libraries but nothing that would fundamentally change the game. We need to develop better predictive methods of in vivo behavior of the delivery systems and move away from 
relying so heavily on in vitro tests that failed us so many times.

What is the key to developing successful collaborative relationships? Beyond the obvious requirement that each partner needs to bring something unique to the table, be it a specialized technique or at least a different perspective, it is important that all collaborators find the project scientifically important and satisfying. For me, it is also helpful to be in the same place geographically when developing successful new collaborations.

What is your philosophy of educating graduate students?

Philosophy is a big word, but on the basic level, I believe in creating a lab environment that allows each student to develop their research skills and tap into their strengths. Students in pharmaceutical sciences must be able to understand concepts and communicate well with people spanning a wide range of fields. It is thus important that the classes students take during their graduate studies reflect that need. I also believe that the PI has a responsibility for the future career success of the students. Because of a disconnect between the way we fund academic research and the job market, there is a surplus of Ph.D. graduates, and the PIs should be mindful of that when deciding how many graduate students to mentor.

What are the challenges facing the pharmaceutical sciences?

Pharmaceutical sciences, both academic and industrial, face numerous challenges. On the academic side, I think that a main challenge for pharmaceutical sciences is to redefine its place in academia. The traditional place in Colleges of Pharmacy is eroding because of the continuing changes in PharmD curriculum and its increasing emphasis on clinical practice. At the same time, many of the important research advances, at least within my field, are coming from scientists at Colleges of Engineering.

What is the place for collaboration with industry in academia? A major portion of academia shows strong disdain for industry and pharmaceutical industry in particular. I think that pharmaceutical scientists, and applied scientists in general, are those that most realize the importance of partnership and collaboration with industry to successfully translate academic findings into practice. The sheer complexity of the regulatory environment and astronomical costs faced in pharmaceutical development make it impossible to succeed without effective partnership with the industry.

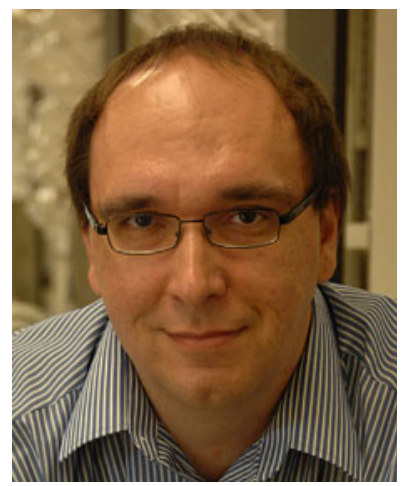

David Oupicky is an Associate Professor of Pharmaceutics in the College of Pharmacy and Health Sciences at Wayne State University in Detroit. He received his M.S. (1993) in Polymer Engineering from Prague Institute of Chemical Technology and his Ph.D. (1999) in Macromolecular Chemistry from the Institute of Macromolecular Chemistry, Academy of Sciences of the Czech Republic. He was a postdoctoral fellow at CRC Institute for Cancer Studies in Birmingham (UK) prior to joining WSU faculty in 2002. He is a member of Karmanos Cancer Institute and adjunct faculty at the Department of Biomedical Engineering, WSU. His research focuses on the synthesis and evaluation of novel stimulus-responsive polymers and nanomaterials for drug and nucleic acid delivery. He is the author of over 70 publications. Dr. Oupicky has been recognized by the Ministry of Education of the Czech Republic Award for Outstanding Young Scientists and by the American Foundation for Pharmaceutical Education Faculty New Investigator award. 